\title{
Computer-Aided Optimization of dc/dc Converters for Automotive Applications
}

\author{
Timothy C. Neugebauer David J. Perreault \\ Massachusetts Institute of Technology \\ Laboratory for Electromagnetic and Electronic Systems \\ Cambridge, MA 02139 USA
}

\begin{abstract}
This paper investigates computer-aided optimization of dc/dc converters, with a focus on converters for dual-voltage automotive electrical systems. A new CAD optimization approach based on Monte Carlo search methods is introduced which allows the design space to be rapidly explored in an automated fashion and the most optimal designs and design approaches to be identified. The optimization approach also allows the effects of variations in design specifications on the cost, weight, and volume of an optimized converter to be readily determined.
\end{abstract}

\section{INTRODUCTION}

The electrical load in automobiles has been growing rapidly for many years, and is starting to exceed the practical capacity of present-day electrical systems. To address this challenge, it is widely agreed that a dualvoltage system is the next evolutionary step for automotive electrical systems. High-power loads will be moved to a new high-voltage bus, while incandescent lighting, electronics, and other loads that benefit from a lower voltage will remain on the currently-used low-voltage bus [1-3].

One widely-considered method for implementing a dual-voltage electrical system is illustrated in Fig. 1. A high-voltage alternator supplies the high-voltage bus, while the low-voltage bus is supplied from the high-

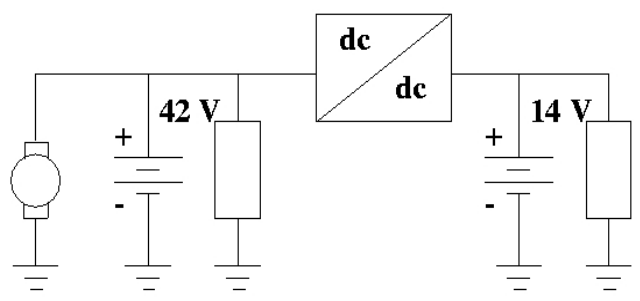

Figure 1 A dual-voltage automotive electrical system. voltage bus via a dc/dc converter. The converter controls the flow of energy between the two buses, and can be used to regulate the energy balance between the high- and low-voltage batteries. In the case where a bidirectional converter is used, either battery may be used to recharge the other if it becomes depleted. These advantages make the dc/dc converter-based system the most desirable of available options from a performance standpoint.

The major practical drawback to the dc/dc converterbased architecture is the relatively high cost of the dc/dc converter, and to a lesser extent its size and weight. This fact, along with the high production volumes associated with automotive components, motivate the optimization of converters for this application. Nevertheless, the task of truly optimizing such a converter is extremely challenging due to the large number of design possibilities that exist and the tight interrelation among many of the design choices. It is also difficult to ascertain a priori how changes in the system-level specifications will affect the cost, weight, and volume of an optimized converter.

In this paper we investigate the computer-aided optimization of power converters for dual-voltage automotive electrical systems. A new Computer-Aided Design (CAD) optimization approach is introduced which allows the design space to be rapidly explored in an automated fashion and the most optimal designs and design approaches to be identified. It also allows the effects of variations in the design specifications on the cost, weight, and volume of an optimized converter to be readily determined, an attribute of particular value 
to the electrical system designer. Section II of the paper reviews some of the existing work on computeraided optimization of power electronics. Section III introduces the approach, describes the structure of a new optimization tool that implements it, and details some of its key elements. Experimental validation of some important models and outputs of the program are also presented. Results demonstrating the use of the new optimization approach are described in Section IV. These include comparison of a computer-optimized design to a conventional design, and illustration of the use of the program to identify both trends in the design space and the effects of variations in the design specification. Finally, Section V draws conclusions and presents an overall evaluation of the approach.

\section{OPTIMIZATION BACKGROUND}

There has been some previous work on computeraided optimization of power electronics. In [4], an optimization routine is coupled with a circuit simulator to select parameters for a power converter filter. The objective function to be minimized is a predetermined function of the filter inductance, capacitance, and output voltage ripple. The circuit simulator ACSL is used to predict the ripple performance of a design, while a deterministic outer loop searches for the objective function minimum.

In [5] the joint optimization of converter structure and control behavior is formulated as a constrained optimization problem and solved using a numerical optimization procedure. The structural objective of the optimization is formulated in terms of converter losses or efficiency, which are based on simplified closedform calculations [6], while the control cost is based on a formulation of the control effort and performance. The authors apply this approach to the design of a buck converter, where the plant design parameters are the converter inductance and capacitance, with other plant parameters fixed. A major disadvantage to this approach is that it requires a very detailed mathematical formulation of the optimization problem.

In [7], computer-aided optimization of a three-phase inverter is considered. The objective function of the optimization is formulated in terms of efficiency or temperature rises. Analytical expressions for converter losses and temperature rises are developed and used for the necessary calculations. The optimization is done via stepped search across two design parameters (gate resistance and gate drive voltage) with all other design choices fixed.

While having distinct aspects, the existing investigations have a number of important characteristics in common. First, in each case, only a very small number of parameters are searched over, meaning that only a small fraction of the entire design space is explored. It is not clear to what extent the optimization techniques and problem formulations extend to a more extensive optimization. Second, all of the techniques consider numerical parameters only, without bridging the gap to actual components. For example, $[4,5]$ search for optimized inductance values, but there is no tie to actual component design or consideration of actual component non-idealities. This makes it difficult to optimize for quantities such as price, weight, and volume, and does not address many of the issues found in an actual design. Finally, all of these investigations focus primarily on the computations and formulation aspects of optimization. However, for a CAD optimization tool to be useful across a large design space and to address component implementation, user interface and data management issues become very important. It is the authors' belief that these issues need to be addressed in a fully functional optimization tool. 


\section{A New Optimization ToOl}

\section{A. Optimization approach}

To address the challenge of optimizing converters for the automotive application, we have developed a CAD optimization tool that is fundamentally different from those developed previously. The optimization program explores the converter design space to identify the converter design or set of designs that best minimizes an objective function that is a weighted sum of component volume, weight, and cost.

To perform an optimization run, the design specifications must first be set, including the power rating, ambient temperature limit, input and output voltage ranges, and input and output electromagnetic interference (EMI) specifications. The user also has control over the region of the design space to be explored. Design variables considered by program include the power stage topology, number of interleaved power stages, switching frequency, inductor ripple ratio, EMI filter topologies and design points, and passive component selection.

A combination of a Monte Carlo system and an expert system is used to choose all the components needed for the converter. A Monte Carlo approach is used in which the program chooses random starting points in the user-defined design space, and designs converters based on these starting points which meet the desired specifications. Some components are not chosen randomly. For example, an expert system approach is used to select the heatsink necessary for the design based on the selected power stage components, their computed losses, and the prespecified operating conditions.

Each converter is designed with specific components of known cost, weight, volume, and operating characteristics. Detailed models are used to accurately predict the system behavior with these components. The output of the program thus consists of converter designs that are composed of available components and have well-defined performance characteristics. After the program designs a large number of converters across the possible design space, the results can be analyzed to determine which converter designs are the most desirable.

\section{B. Program Structure}

The converter specifications and design variables presently incorporated in the optimization program are those considered appropriate for the automotive power conversion application. For example, only converter topologies and components suitable for this application are included. Nevertheless, the optimization program is object-oriented, and its structure is quite general, so the code itself can be readily modified to admit a wide variety of other specifications and design variables, and can easily be adapted to other applications.

The optimization program consists of five main elements, as illustrated in Fig. 2. These elements are the control loop, the design algorithm, the device models, the database, and the user interface. The control loop is the element that guides the search of the

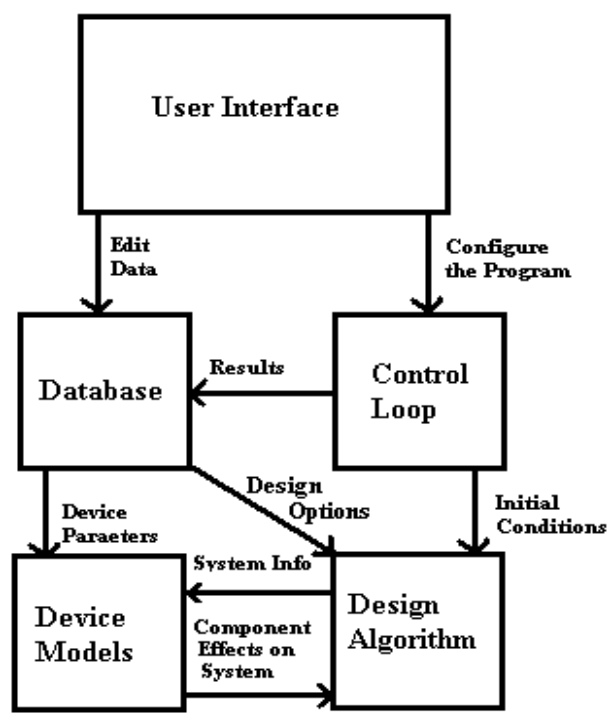

Figure 2 The structure of the optimization program. 
design space. It randomly chooses the design starting points to be used for each iteration, and passes these variables to the design algorithm.

The design algorithm uses variables generated by the control loop and previously generated results to design the converter subsystems. The design algorithm ensures that any designs saved are valid; as soon as a design proves to be invalid the design algorithm stops and the control loop will generate new design variables.

To generate the information necessary for the design of the converter, the design algorithm calls on device models of the converter components. Each of these models consists of code that describes the necessary aspects of a component's design and use. For example, the model for an inductor contains all the code necessary to design the inductor, as well as to predict its behavior (such as power losses, temperature rises, etc.). If a component can not be found to adequately meet one of the specifications, then the iteration is declared invalid and the control loop chooses another point in the design space to analyze.

There are three main types of databases used in the CAD program. The part list databases store information about all the parts that can be used by the optimization program. Configuration databases store the variables used to define the design space and set design specifications. The results databases store the components used and pertinent information about each valid converter found during the design space search.

The user interface has a number of functions. It allows one to edit the properties of all the components in the database, to set up the converter specifications and design space, and to view the optimized designs and other information generated by the program.

\section{Optimization and Program Implementation}

The optimization process used by the program is illustrated in Fig. 3. The control is divided into an inner and outer control loop in order to accelerate the optimization process. The outer loop randomly chooses the design variables that are needed to construct the power stage of the converter. The power stage design algorithm then uses these values as initial conditions and determines essential values of the design, chooses components to ensure proper operation of the converter, and makes sure a valid power stage is achieved. If a valid design is found the inner loop and filter design algorithm will attempt to design input and output filters that will meet the EMI specifications. Thus, for each iteration of the outer control loop a power stage will be design and the inner loop will find input and output filters optimized for that power stage.

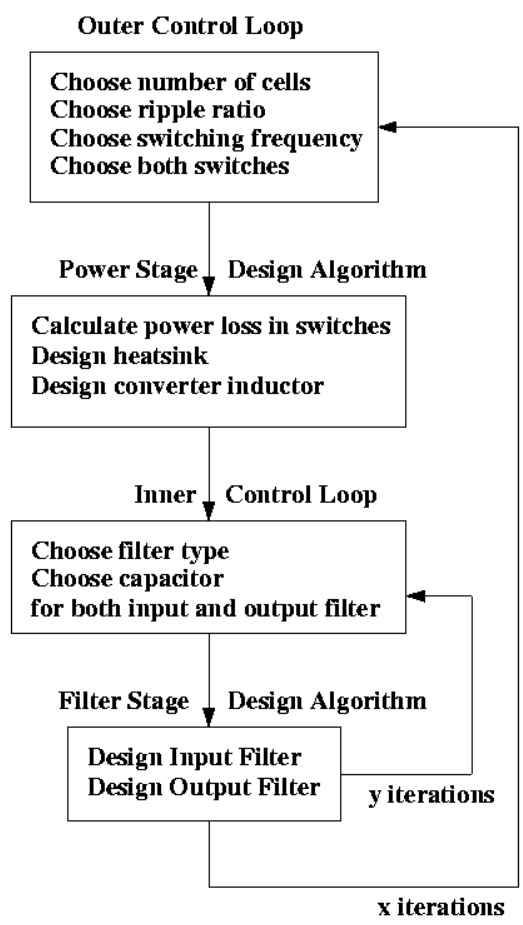

Figure 3 The relationships between the control loop and the design algorithm. The outer loop calls the power stage design algorithm. After the power stage is designed the inner control loop runs and the filter stages are designed. The inner control loop iterates $y$ times and the outer control loop iterates $x$ times. 
Once a design is successfully completed, the program will calculate the total cost, weight and volume of the valid design and save this record in a database. This information can then be used to rank the design and after a thorough examination of the design space the most optimal converter can be found.

\section{Subsystem Design Example}

For every major component used in the converter there are subroutines that accurately model the component and make appropriate design decisions for it. For example, consider the subroutine used to design the converter power stage inductors.

The routine takes as input the desired inductance and operating range and utilizes the database of available inductor cores and their properties. The routine's output includes the chosen core, the number of turns and wire gauge needed, the power dissipated in the core, and its temperature rise.

The potential inductor cores are ranked beforehand based on their suitability; for example the cores may be pre-ranked on cost. The program then finds the lowest ranked core that is suitable for the design.

The first step in the design to find the number of turns needed and the resulting flux density that will be induced in the core. If this flux density is high enough to saturate the core, the core is rejected as a candidate.

The next step is to determine the wire size. The turns of wire must fit within the window area of the core. The program finds the largest diameter wire that will fit within the window area. The program verifies that this wire size will result in a maximum current density, $\mathrm{J}_{\max }$, of less then $3000 \mathrm{~A} / \mathrm{cm}^{2}$ in the wire. If not, the core is rejected as a candidate.

Because of the significant dc and ripple components in the current waveform, there will be significant winding and core losses. The winding loss for the inductor, including skin and proximity effect, is computed using the models developed in [8].

To calculate core loss, the ac flux swing in the core is approximated as a sinusoid and the core loss is calculated using the models provided by Ferroxcube/Phillips [9].

Based on the sum of winding and core losses, the centerpost temperature rise of the core can be estimated as the product of the power dissipation and the thermal resistance of the core. If the temperature rises above the maximum allowable temperature, the inductor design is rejected, otherwise the program accepts the design of the inductor.

\section{E. Program Validation}

In order for the optimization program to be useful for exploring the design space, it must be able to generate realistic converter designs and be able to accurately predict operating characteristics such as losses, temperature rises, and EMI performance. A great deal of emphasis has been placed on validating the modeling and predictions made by the optimization program for this reason. One example of experimental validation of the program models is illustrated in Table 1. The heat sink and inductor core temperature rises of an experimental prototype were compared with the predictions for that design made by the optimization program models. The close match between these values indicates that the optimization program models

\begin{tabular}{|l|l|l|}
\hline Component & $\begin{array}{l}\text { Measured } \\
\text { Temperature } \\
\text { Rise }\end{array}$ & $\begin{array}{l}\text { Calculated } \\
\text { Temperature } \\
\text { Rise }\end{array}$ \\
\hline Converter Inductor & $13.3^{\circ} \mathrm{C}$ & $13.7^{\circ} \mathrm{C}$ \\
\hline Heat Sink & $11.9^{\circ} \mathrm{C}$ & $12.3^{\circ} \mathrm{C}$ \\
\hline
\end{tabular}

Table 1 Comparison of inductor core and heat sink temperature rises of an experimental prototype to those predicted by the optimization program. Values are for a specific converter operating at $500 \mathrm{~W}$ in $25{ }^{\circ} \mathrm{C}$ ambient temperature; predictions and measurements at rated power and ambient temperature $(1000 \mathrm{~W}, 105$ ${ }^{\circ} \mathrm{C}$ ) would be higher. 
accurately predict both the temperature rise and power loss for these elements.

\section{RESUlts}

The optimization program generates converter designs and ranks them based on a specified weighted sum of component cost, weight, and volume. The results can then be used to identify converter designs that best meet the specified objective function or to study trends within the design space. To illustrate the use of the program for these purposes, we consider example results for the volume optimization of $\mathrm{dc} / \mathrm{dc}$ converters meeting the specification of Table 2. (These specifications are typical of what might be required of a power converter for a future dual-voltage automotive electrical system.) Note that volume is computed as the sum of the volumes of the three-dimensional outlines of the individual components. For example, the volume of a rectangular heat sink is computed as the volume of the smallest box in which it would fit.

A prototype power converter meeting the specifications of Table 2 was designed and constructed. The prototype converter, which was developed using a reasonable design approach but was not explicitly optimized for volume, has a component volume of approximately $1000 \mathrm{~cm}^{3}$. For comparison, consider that the lowest-volume design generated by the optimization program has a component volume of only $210 \mathrm{~cm}^{3}$. This illustrates the kind of results that are achievable using the CAD optimization approach over what is easily achieved by hand.

The optimization program can also be used to

\begin{tabular}{|l|l|}
\hline \multicolumn{1}{|c|}{ Input Voltage range } & $33 \mathrm{~V}-53 \mathrm{~V}$ \\
\hline Output Voltage range & $11 \mathrm{~V}-16 \mathrm{~V}$ \\
\hline Power Rating & $1000 \mathrm{~W}$ \\
\hline Ambient Temperature & $105^{\circ} \mathrm{C}$ \\
\hline EMI Specifications & $\mathrm{SAE} \mathrm{J1113/41} \mathrm{Class} \mathrm{1}$ \\
\hline
\end{tabular}

Table 2 Design specifications for a power converter in a dual-voltage automotive electrical system. investigate trends in the design space. For example, Fig. 4 illustrates how the volume of a volumeoptimized converter varies with power rating. To obtain this data, the optimization program was used to identify the lowest-volume design across the design space (which includes the switching frequency of the converter) for converters rated at 250, 500, 750, and $1000 \mathrm{~W}$ (with other specifications as in Table 2). From this, one finds an approximately (incrementally) linear relationship between converter volume and power rating which can be represented as

$$
\text { Volume }\left(\mathrm{cm}^{3}\right) \approx 0.18 \cdot \text { Power }(W)+29.88
$$

over this range. Similar evaluations of converter weight, cost, and other attributes can also be made. This type of result is difficult to obtain by conventional means, and is of particular value to the automotive electrical system designer who must trade converter size, weight, and cost against other system-level design considerations.

The computer-aided optimization approach is also useful for studying the sensitivity of the optimized converter design to changes in design specifications. For example, the relationship between the volume of a volume-optimized converter and its power rating is dependent upon the ambient temperature specification of the converter. Fig. 5 shows this temperature dependence, calculated using the same process as

Volume vs. Power Rating

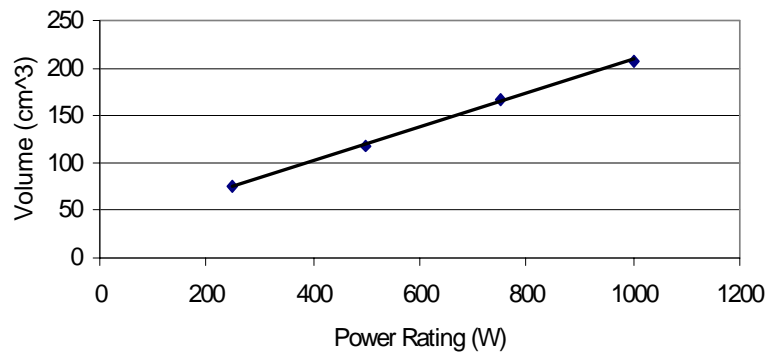

Figure 4 Relationship between the volume of the volume-optimized converter and its power rating. 


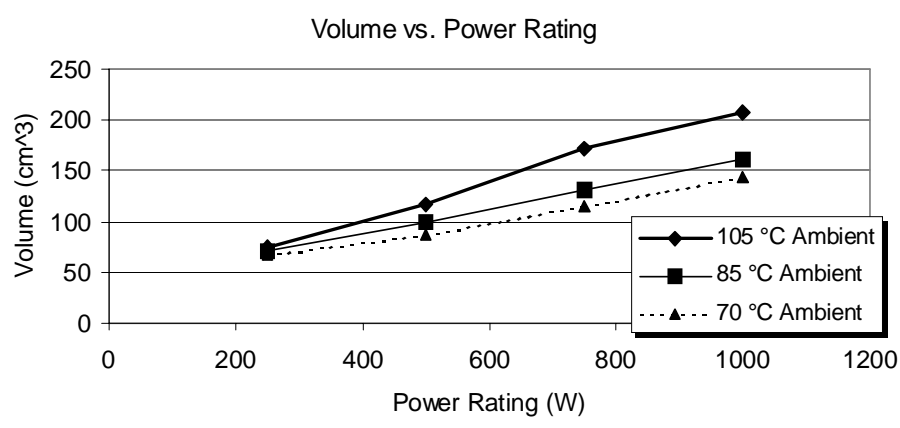

Figure 5 The volume of the smallest converter at various power ratings for three different ambient temperatures.

above across different ambient temperature requirements. The sensitivity of converter size to increases in ambient temperature rating becomes pronounced at higher temperature and power levels. This is expected since temperature rise margins and component availability become impinged at high temperatures and power levels.

Other design requirements (such as EMI specifications) also have a strong effect on the optimized converter design. These sensitivities can be much more easily explored using the proposed CAD optimization approach than is possible by conventional means.

\section{CONCLUSIONS}

Power converters for dual-voltage automotive electrical systems will be manufactured in high production volumes under tight cost, size, and weight constraints. To address the challenge of designing converters for this application, this paper investigates computer-aided optimization of $\mathrm{dc} / \mathrm{dc}$ converters. A new CAD optimization approach based on Monte
Carlo search methods is introduced which allows the design space to be rapidly explored in an automated fashion and the most optimal designs and design approaches to be identified. The output of the program consists of converter designs that are composed of available components and have well-defined performance characteristics. The program also allows the effects of variations in design specifications on the cost, weight, and volume of an optimized converter to be readily determined.

\section{REFERENCES}

[1] J.G. Kassakian, H.-C. Wolf, J.M. Miller, and C.J. Hurton, "The Future of Automotive Electrical Systems," Proc. IEEE Workshop on Power Electronics in Transportation, Hyatt-Regency Hotel, Dearborn, MI, Oct. 24-25, 1996, pp. 3-12.

[2] J.M. Miller, D. Goel, D. Kaminski, H.-P. Schöner, and T.M. Jahns, "Making the Case for a Next Generation Automotive Electrical System," International Congress on Transportation Electronics Convergence '98, pp. 4151 .

[3] J.G. Kassakian, H.-C. Wolf, J.M. Miller, and C.J. Hurton, "Automotive Electrical Systems circa 2005," IEEE Spectrum, August 1996, pp. 22-27.

[4] J.J. Jeyappragash, T.V. Sivakumar, and V.V. Sastry, "Object Oriented Modeling, Simulation and Optimization of Power Electronic Circuits," 1996 IEEE Power Electronics Specialists Conference, pp. 581-585.

[5] C. Gezgin, B.S. Heck, and R.M. Bass, "Simultaneous Design of Power Stage and Controller for Switching Power Supplies," IEEE Transactions on Power Electronics, vol.12, no.3, May 1997, pp. 558-566.

[6] A. Reatti, "Steady-state analysis including parasitic components and switching losses of buck and boost DCDC PWM converters under any operating condition," International Journal of Electronics, 1994, Vol. 77, No. 5, pp. 679-701.

[7] F. Blaabjerg and J.K. Pedersen, "Optimized design of a complete three-phase PWM-VS inverter," IEEE Transactions on Power Electronics, vol.12, no.3, May 1997, pp. 567-577.

[8] B. Carsten, "High Frequency Conductor Losses in Switchmode Magnetics," Power Conversion and Intelligent Motion Magazine (PCIM), Nov. 1986, pp. 3446.

[9] Ferrite Material and Components Catalog, $8^{\text {th }}$ Ed., Philips Components, Discrete Product Division, Riviera Beach, FL. 